\title{
Adaptation et évolution des techniques de traitement de sol en matière de protection de l'environnement
}

\author{
Adaptation and evolution in techniques of ground treatment \\ for the protection of the environment
}

\author{
Annette ESNAULT \\ SIF BACHY*
}

Rev. Franç. Géotech. nº 60, pp, $27-40$ (juillet 1992)

\section{Résumé}

La protection de l'environnement est une préoccupation majeure qui touche la plupart des secteurs industriels.

L'implication dans ce domaine d'une entreprise spécialisée en géotechnique est double. Elle concerne d'une part l'impact sur le milieu naturel des produits utilisés dans la profession, plus particulièrement des produits d'injection et d'autre part, la mise en place à titre préventif ou curatif de moyens susceptibles de résoudre des problèmes liés à la protection de l'environnement.

Dans cette optique, la société BACHY a mis au point ces dernières années des coulis d'injection entièrement minéraux. La protection du milieu naturel et la réhabilitation des zones polluées demandent la maîtrise de techniques très variées telles que les parois étanches pour l'exécution d'enceintes de confinement, les parois drainantes pour la récupération de gaz et de lixiviat, les injections, le jetgrouting et les méthodes de deep-mixing pour l'exécution de fonds étanches et l'inertage en place des déchets.

L'article met l'accent sur les derniers développements tant au niveau de coulis d'injection que de l'approche adoptée pour la formulation de coulis de parois.

\footnotetext{
Abstract

Environmental protection is one of the major concerns of industrial sectors. The involvement of a specialist geotechnical company is twofold : firstly, concerning the effects on the natural environment of the products used in the geotechnical profession, in particular the products of grouting ; and secondly the design and implementation of methods that will solve some of the problems linked with environmental protection.

As a result $\mathrm{BACHY}$ has developped, over a period of several years a range of grouts that are entirely mineral based.

Furthermore, the protection of the natural environment and the regeneration of polluted zones requires a mastery of a variety of techniques : impermeable plastic concrete walls or slurry walls for the construction of watertight cut-offs, draining walls for the recovery of gas and leachate, grouting, jet-grouting and deep mixing methods for the construction of impermeable barrier layers and the in situ neutralisation of waste products.

The article will present the latest developments on grouts and the approach adopted for the formulation of slurries.
} 


\section{INTRODUCTION}

De nombreux cas de contamination de sol dus au stockage ou au rejet de déchets provenant d'activités industrielles sont apparus au cours des dernières décennies.

Ces pollutions peuvent avoir des conséquences extrêmement variées sur le milieu naturel puisqu'elles représentent une menace pour les eaux de surface et les aquifères.

La situation est telle qu'actuellement on ne peut pas ne pas parler d'environnement. Il existe une réelle sensibilisation au problème, que ce soit à travers les médias ou les mouvements écologiques quand un cas de pollution est suspecté ou déclaré. La réglementation a également beaucoup évolué et on tend vers des choix et des définitions de sécurité maximale en matière d'environnement. A ce niveau, tous les secteurs industriels sont concernés. Les entreprises relevant de la géotechnique n'échappent pas à cette règle.

Parallèlement certaines techniques couramment utilisées pour des traitements * classiques " de sol peuvent être adaptées pour apporter des solutions efficaces à la protection et la sauvegarde de l'environnement.

\section{GÉNÉRALITÉS}

\section{SUR LA SITUATION ACTUELLE}

Les sources existantes susceptibles d'entraîner une pollution sont très variées.

Elles sont représentées notamment par le stockage de déchets de diverses industries et par le rejet accidentel ou systématique de produits toxiques vers le milieu naturel.

Les différentes natures de produits polluants rencontrés sont le reflet de cette variété : hydrocarbures, solvants, métaux lourds, acides minéraux ou organiques, etc...

Les mesures à mettre en place pour minimiser ou annuler les risques de pollution dépendent bien sûr du niveau auquel le problème est abordé. Il en existe donc de deux types:

- des mesures préventives ;

- des mesures curatives.

Les mesures préventives concernent le choix des produits utilisés par l'industriel et leur mode d'élimination, ceci de façon à minimiser les risques encourus. Le choix s'effectue selon des critères parfaitement définis dans le cadre de lois régissant les conditions de fonctionnement d'un secteur industriel particulier. Elles consistent aussi à se préparer à l'éventualité d'un accident en prévoyant, dès la conception du projet, des moyens visant à en limiter les conséquences. On peut citer comme exemple la mise en place d'un dispositif de confinement autour de la zone à risque.

Les mesures curatives consistent à éliminer la source même de pollution ou à limiter son expansion. Cela peut être réalisé en appliquant des techniques de décontamination de sol (inertage in situ, extraction des contaminants, etc.) associées à des techniques de confinement et de drainage.

On retrouve donc les deux niveaux auxquels l'entreprise BACHY se trouve impliquée;
- réflexion au sujet de l'impact sur l'environnement des produits qu'elle utilise et plus particulièrement des produits d'injection ;

- mise en place au niveau préventif ou curatif de moyens susceptibles d'apporter une solution au problème de contamination de sol.

\section{LES INJECTIONS : COULIS MICRON-S}

Les injections sont utilisées à des fins d'étanchement ou de consolidation d'un sol.

Elles consistent à faire pénétrer dans les vides du terrain à traiter (fissure ou vide intergranulaire) un coulis sous forme liquide (gel ou résine) ou sous forme de suspension (ciment ou autre matériau pulvérulent). Le coulis ainsi injecté assurera après prise les propriétés mécaniques ou hydrauliques recherchées.

Quand on réalise une intervention sur un milieu naturel, en l'occurrence un sol, on peut s'interroger sur les éventuelles conséquences d'un tel traitement à partir du moment où l'on modifie l'état d'origine du milieu.

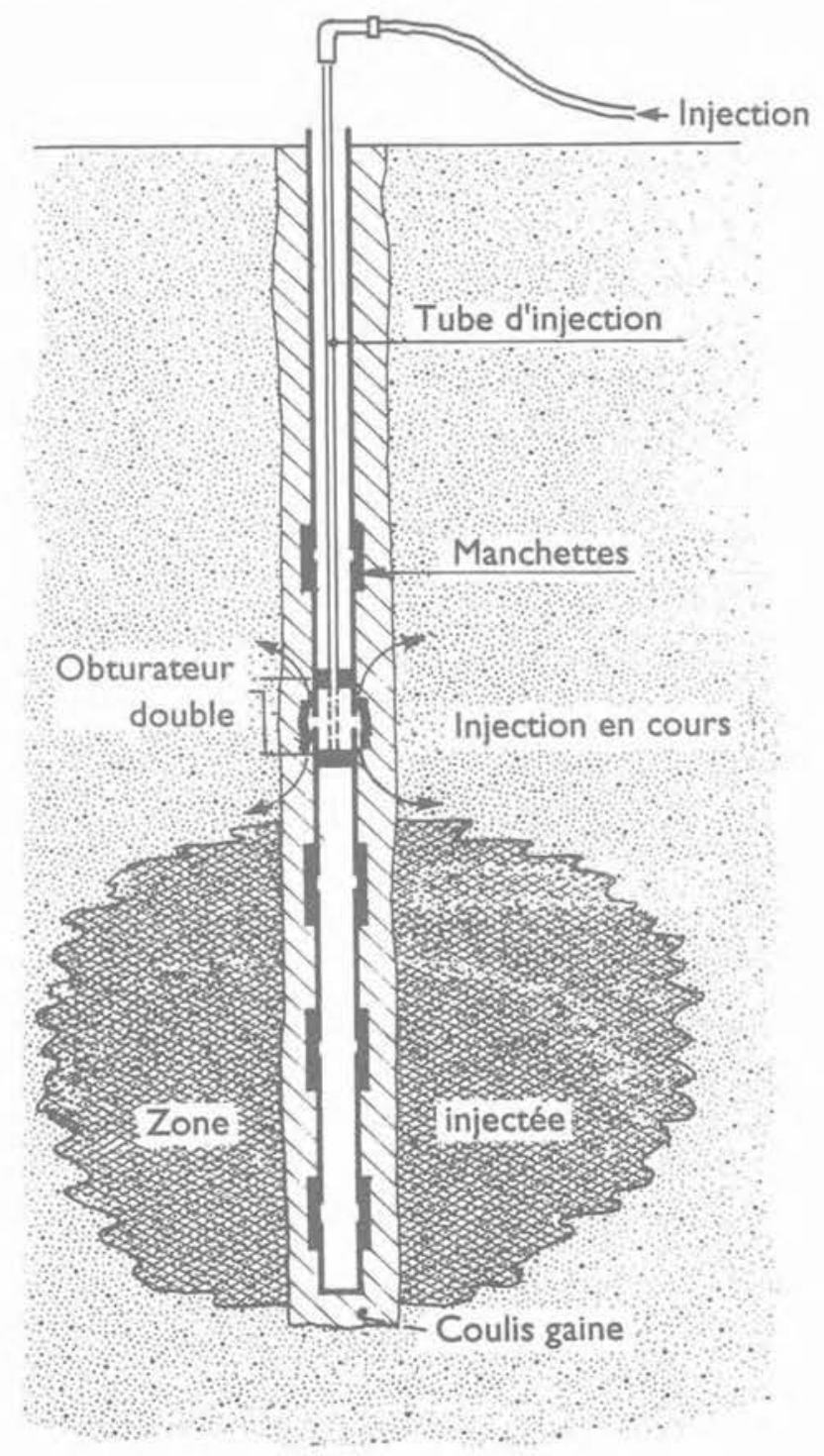

Fig. 1. - Principe de l'injection.

Fig. 1. - Injection process. 
Le choix des matériaux d'injection est donc essentiel et il est nécessaire d'utiliser des matériaux n'affectant pas l'intégrité du milieu naturel. $\mathrm{Ce}$ choix devient primordial quand on travaille dans des environnements sensibles, à proximité d'une nappe phréatique par exemple. Il a donc fallu mettre au point ces dernières années des coulis d'injection minéraux répondant à ce souci.

La propriété gouvernant la mise en place de ces coulis est celle de "pénétrabilité ".

Les études ont donc particulièrement porté sur l'augmentation des performances des coulis de ciment de la famille des C3S et sur le développement d'un coulis minéral pour l'injection de terrains granulaires fins, le MICRON S

Ce coulis est composé de microsilice et de chaux. La prise entre les deux composants s'apparente à une réaction pouzzolanique conduisant à la génération de silicates de calcium hydratés $(\mathrm{CSH})$ sans formation de produits secondaires.

L'extrême finesse des matériaux de base rend le coulis parfaitement adapté au traitement de terrains granulaires de très faibles perméabilités $\left(<10^{-5} \mathrm{~m} / \mathrm{s}\right)$ (voir fig. 2 et 3 ).

Des essais de lixiviation à l'eau réalisés sur le coulis durci ont montré que la teneur en éléments minéraux passant en solution restait inférieure à la teneur admissible pour les eaux potables (voir fig. 4).

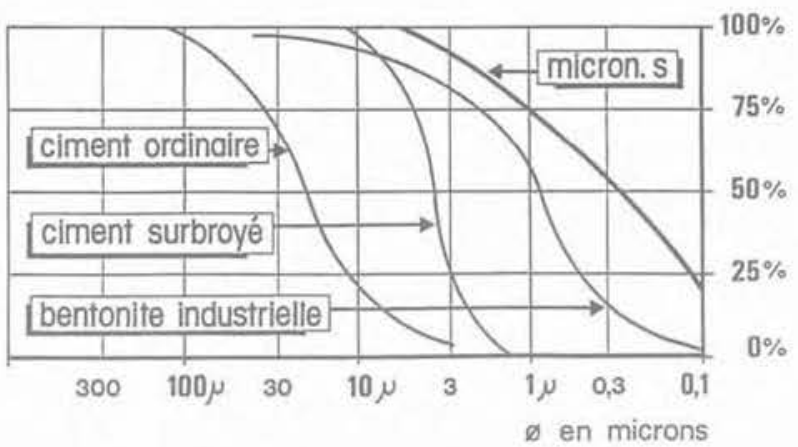

Fig. 2. - Analyses granulométriques. Fig. 2. - Grain size distribution.

pénétrabilité du Dg5 du coulis

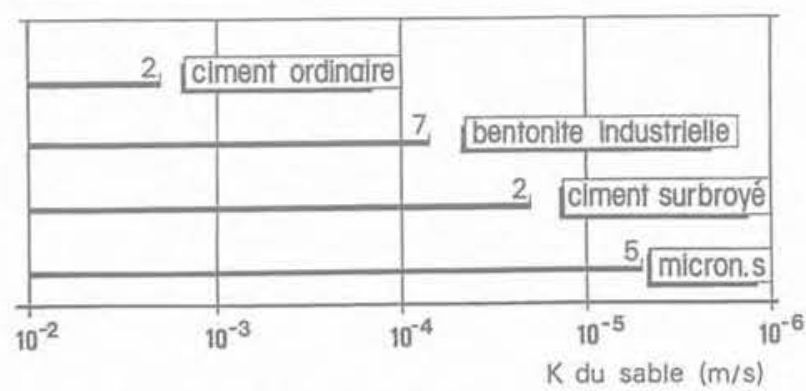

Fig. 3. - Pénétrabilité théorique dans du sable. Fig. 3. - Theoritical penetrability in sand

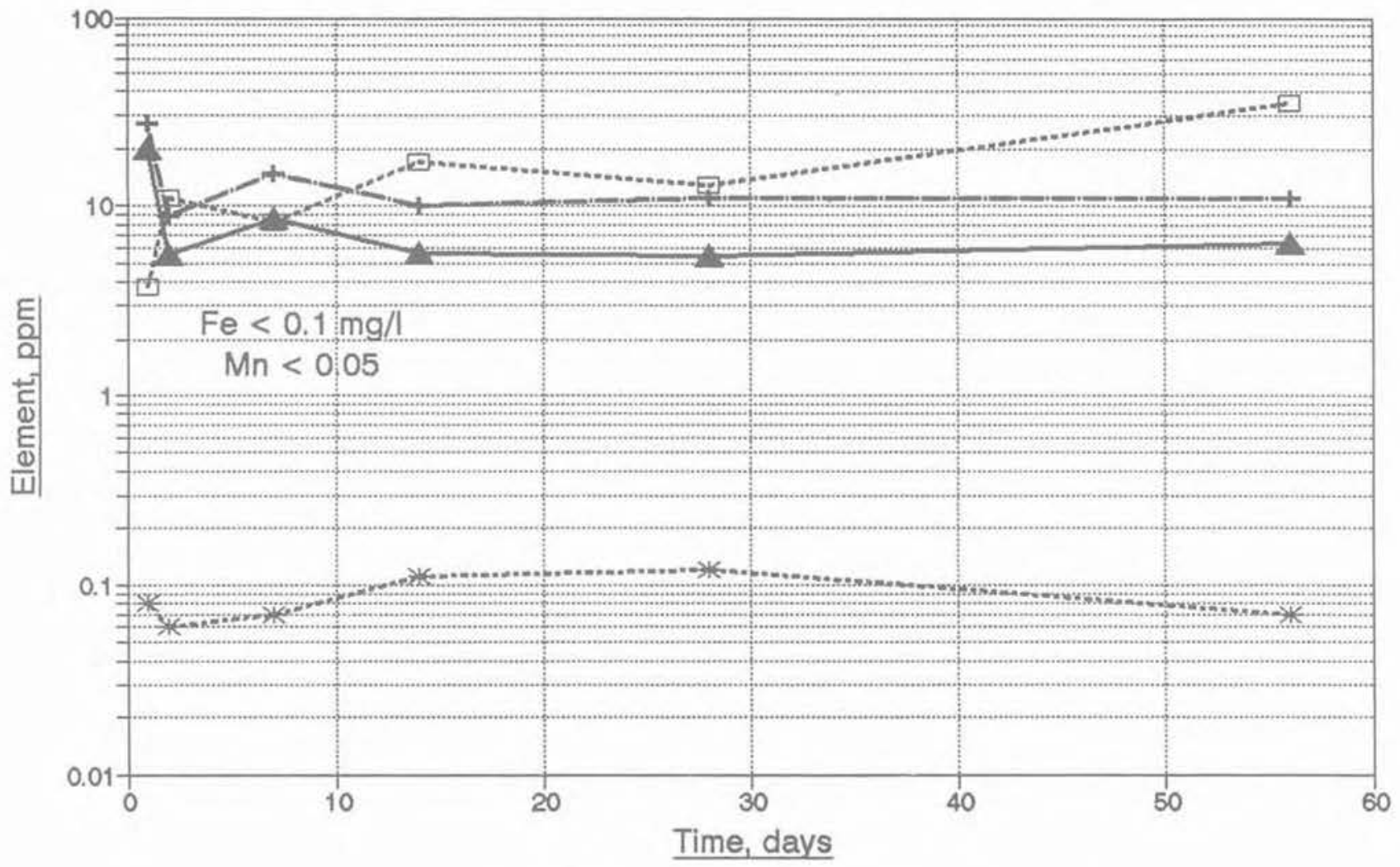

$\mathrm{Na}+\cdots \mathrm{K} \cdots *-\mathrm{Mg} \cdots \mathrm{Ca}$

Fig. 4. - MICRON S - Test de lixiviation sur coulis. - Fig. 4. - MICRON S - Leaching from neat grout. 


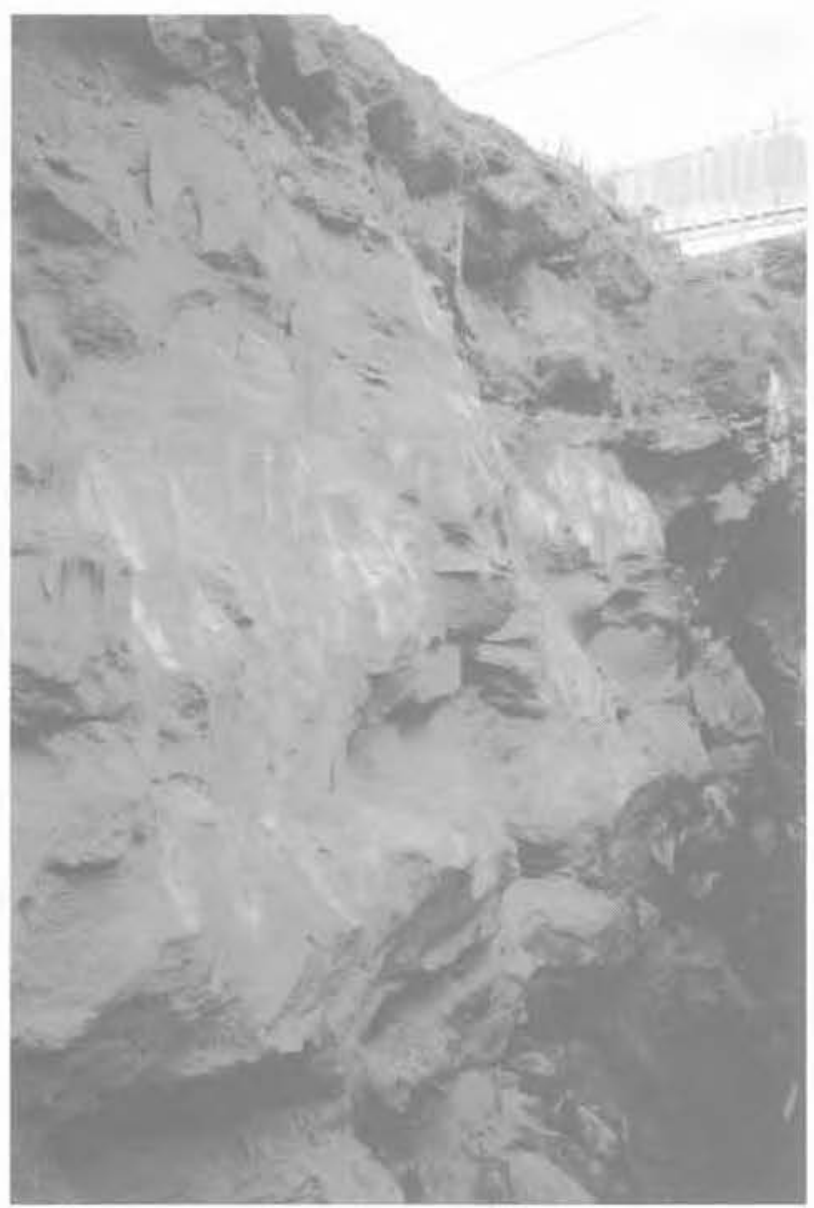

Fig. 5. - MICRON S - Nantes - Zones injectées. Fig. 5. MICRON S - Nantes - Grouted areas.

Cette propriété le rend donc particulièrement intéressant pour une utilisation en milieu sensible.

Cette notion de "coulis écologique s est devenue l'un des fils conducteurs des recherches futures.

\section{GÉOTECHNIQUE ET ENVIRONNEMENT}

Différentes techniques utilisées en géotechnique peuvent être adaptées pour résoudre des problèmes liés à la protection et la sauvegarde de l'environnement.

Elles concernent principalement les écrans horizontaux ou verticaux d'étanchéité, les parois drainantes et les traitements d'inertage in situ de sol pollué.

4.1. Ecran vertical d'étanchéité destiné à limiter l'expansion d'une pollution en confinant la zone concernée.

Il peut être réalisé selon différentes techniques en fonction du type de terrain et du problème rencontré.

Dans le cadre de travaux provisoires et de caractère très urgent, il peut être réalisé par congélation soit du terrain en aval de la zone polluée, soit directement de la zone contaminée. Cette opération demandera un traitement d'entretien souvent assez lourd tant que la source de pollution n'aura pas été éliminée.
Un traitement permanent peut être réalisé par l'intermédiaire de paroi et/ou par injection. Les deux techniques peuvent, dans certains cas, être complémentaires.

En fonction des critères hydrauliques et mécaniques auxquels la paroi aura à répondre, elle peut être réalisée au coulis, au béton plastique ou au coulis avec l'incorporation d'une membrane plastique.

Les perméabilités atteintes varient de $10^{-7}$ (coulis standard) à $10^{-13} \mathrm{~m} / \mathrm{s}$ (membrane plastique).

Une paroi en béton plastique est perforée sous boue bentonitique et bétonnée avec le mélange béton plastique mis en place par tube plongeur. Généralement, une paroi de ce type a une épaisseur voisine de $0.60 \mathrm{~m}$ à $1 \mathrm{~m}$ et est faite par panneaux alternés. Les joints sont obtenus par grattage des panneaux primaires. Pour les bétons résistants classiques, les joints courants sont réalisés avec des tubes joints alors que les joints étanches font appel au système de coffrage CWS qui permet la mise en place de bandes * waterstop » entre les panneaux.

La paroi au coulis peut être réalisée en continu ou par panneaux alternés. Son épaisseur usuelle est comprise entre $0,50 \mathrm{~m}$ et $0,80 \mathrm{~m}$.

Le principe d'exécution est illustré par le schéma $n^{\circ} 6$. Les profondeurs de ces parois peuvent dépasser $60 \mathrm{~m}$.

Plusieurs types de matériel d'excavation sont disponibles en fonction des profondeurs à atteindre et de la dureté du terrain :

- excavation à la pelle rétro jusqu'à des profondeurs d'environ $15 \mathrm{~m}$ et des terrains \& mous » ;

- excavation à la benne, pour des profondeurs supérieures à $15 \mathrm{~m}$ et des terrains très variés comme alluvions sablo-graveleuses, éboulis, roches sédimentaires tendres, etc. ;

- excavation à la haveuse, pour les très grandes profondeurs et des terrains rocheux durs à très durs ( $R c$ jusqu'à $200 \mathrm{MPa}$ ).

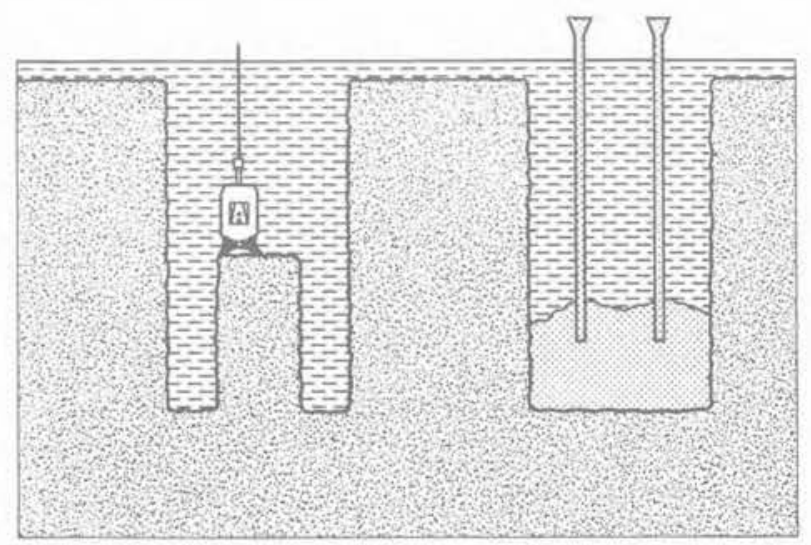

Panneau en cours

de perforation

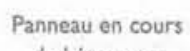

de bétonnage
Fig. 6. - Schéma d'exécution d'une paroi.

Fig. 6. - Construction scheme of a diaphragm wall. 


\subsection{Paroi drainante}

Souvent associée à un écran vertical d'étanchéité, elle est destinée au drainage de gaz ou de liquides pour les diriger vers un système de collecte.

La réalisation d'un dispositif de drainage peut être obtenue par l'installation d'un réseau de drains forés ou par un système de tranchées drainantes permettant de couper et capter les alimentations potentielles a en amont $»$.

La paroi drainante, dérivée de la technique des parois moulées mais avec l'utilisation d'un fluide spécial d'excavation biodégradable à la place de la boue bentonitique habituelle, permet la réalisation de véritables « coupures drainantes » continues de profondeur pouvant atteindre plusieurs dizaines de mètres (voir schéma $n^{\circ} 7$ ).
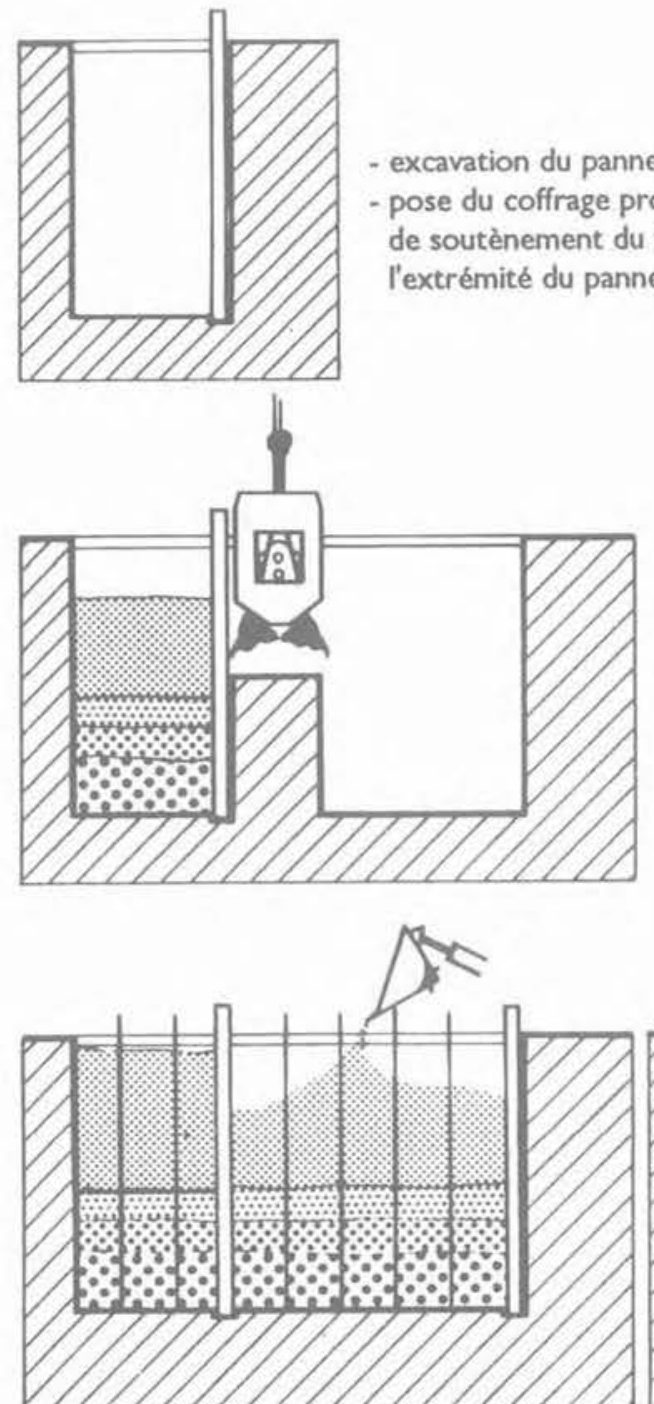

- pose d'un coffrage provisoire de soutènement du filtre à l'extrémité du panneau $n+1$ - mise en place du massif filtrant dans le panneau $n+1$
Le massif filtrant doit avoir une perméabilité suffisante par rapport à celle du terrain pour obtenir un rabattement maximum dans la paroi et une granulométrie telle qu'il se comporte comme un filtre vis-à-vis des grains du terrain pour éviter un colmatage à long terme.

Le filtre peut être éventuellement un filtre multicouches de granulométries différentes ou un filtre composite, par exemple nappes de géotextile au contact du terrain et massif drainant en gravier de perméabilité élevée.

Il peut aussi comporter un ou plusieurs tubes de collature en fond de paroi.

Le fluide d'excavation utilisé est une solution collödale d'un bio-polymère dans l'eau présentant un comportement pseudo-plastique à la préparation et retrou-

\section{MISE EN OEUVRE}

\section{Phases d'exécution d'une paroi drainante}

- mise en place du massif filtrant dans le panneau $n$

- excavation du panneau $n+1$

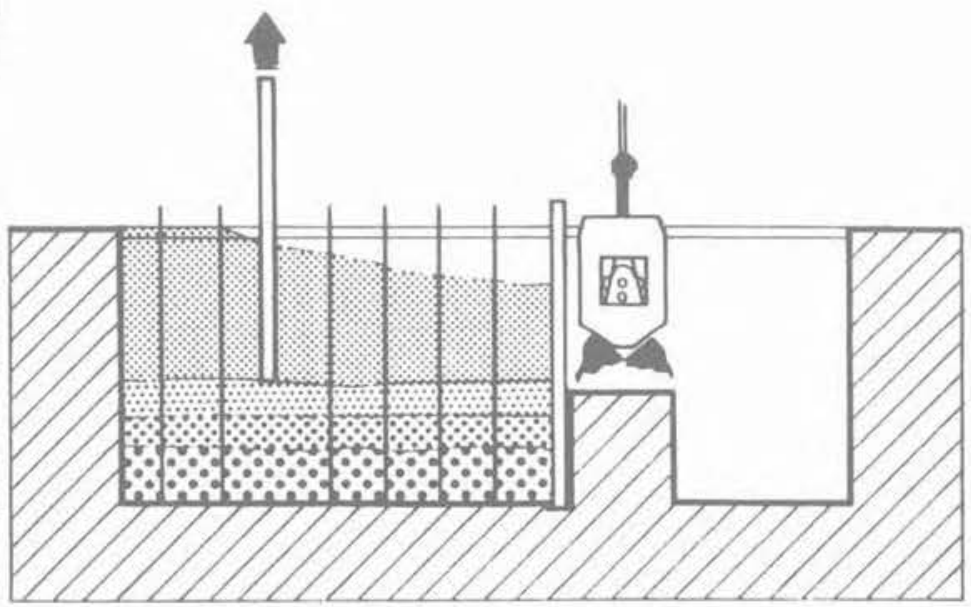

- excavation du coffrage du panneau n

- excavation du panneau $n+2$

Fig. 7. - Phase d'exécution d'une paroi drainante.

Fig. 7. - Construction scheme of a drainage trench 
vant une fluidité voisine de celle de l'eau au bout d'un certain temps par suite de phénomènes de dégradation enzymatique contrôlée. Il est ainsi possible d'éliminer très facilement du massif filtrant la boue ainsi liquéfiée. La « durée de vie », variable suivant les produits, la température, etc. peut être réglée de quelques jours à plusieurs semaines par adjonction d'inhibiteurs.

\subsection{Etanchéité horizontale}

Différentes techniques sont disponibles, parmi lesquelles on peut citer;

- l'injection, technique utilisée pour l'exécution de radier injecté étanche; ce procédé dérive de celui abordé précédemment au chapitre 3 ;

- la mise en place de géomembrane, destinée à la réalisation de fond étanche de zone de stockage de déchets ; cette technique est très connue et documentée ;

- et le jet mix.

Le jet mix est un procédé de traitement des sols par mélange in situ par voie hydraulique dont la conception permet d'en étendre le domaine d'application à tous les terrains meubles, quelles que soit leur compacité ou leur hétérogénéité.

Le jet mix fait appel aux trois phénomènes distincts suivants intervenant indépendamment ou en combinaison :

- une déstructuration des terrains en place sous l'effet hydrodynamique d'un jet à très grande vitesse ;
- une extraction d'une partie des éléments constitutifs du sol en place;

- une incorporation de matériaux d'apport sous la forme d'un jet de coulis de composition adaptée au résultat recherché.

Le jet mix permet la réalisation dans les terrains meubles des ouvrages les plus divers, combinaison des deux formes de traitement élérnentaire que sont la colonne et le panneau (voir fig. 8). L'exécution de colonne jointive permet la réalisation de radier étanche.

\subsection{Traitements in situ}

Il s'agit de décontaminer en place une zone polluée en appliquant une technique destinée à réduire ou à supprimer la mobilité des espèces chimiques présentes.

Cela peut être réalisé soit en transformant les produits concernés en un produit moins toxique, soit en les solidifiant en une masse inerte.

Ce genre de traitement s'effectue par l'intermédiaire de techniques d'injection, de deep-mixing ou de jet mix, en adaptant le type de matériau décontaminant au type de polluant.

Le deep-mixing est une méthode de traitement des sols par mélange in situ avec des agents le plus souvent du type liant hydraulique. Le mélange est réalisé par des outils malaxeurs.

Le procédé col mix est une méthode brevetée de traitement des sols par mélange mécanique in situ dont la conception permet la réalisation de colonnes de sol stabilisé et compacté dans les terrains meubles (voir fig. 9).

\section{IET MIX. Réalisation de colonnes}

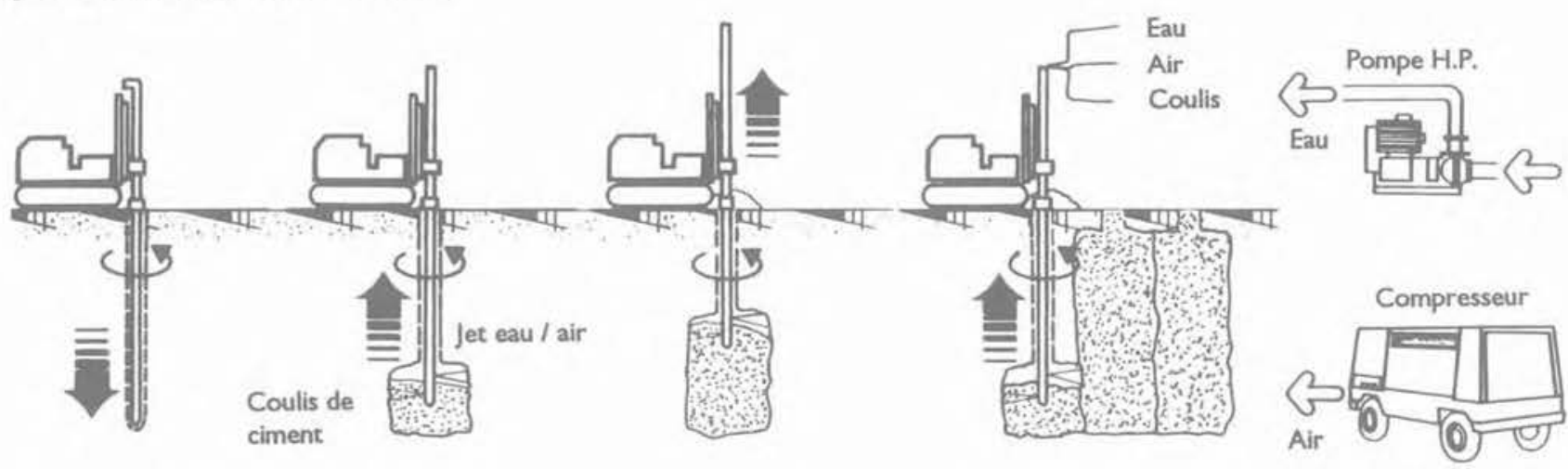

IET MIX. Réalisation de panneaux
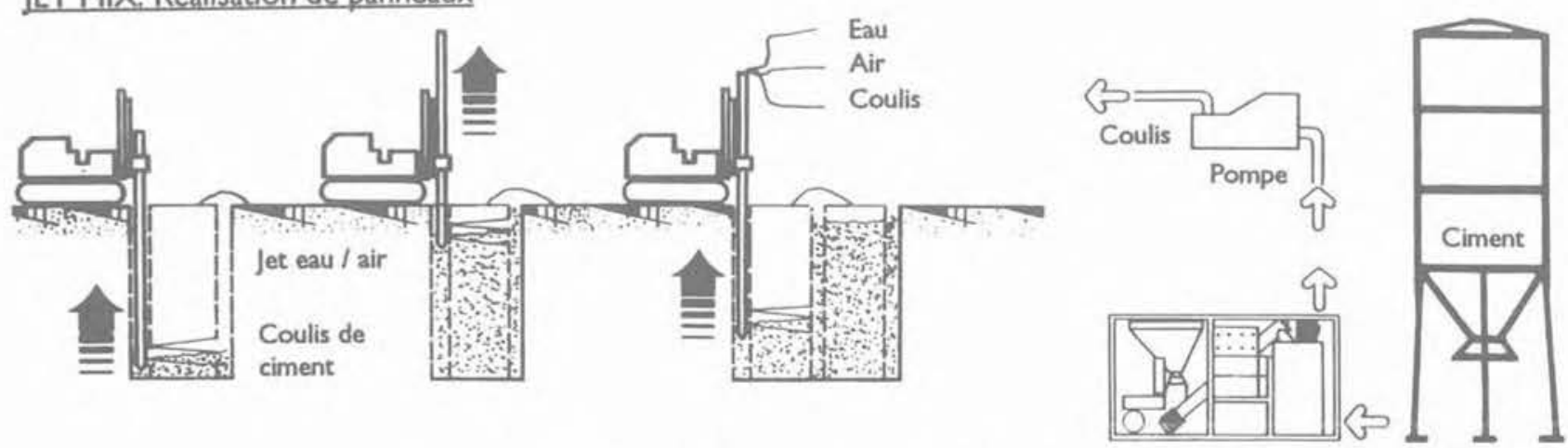

Fig. 8. - Procédé jet mix. - Fig. 8. - jet mix process. 


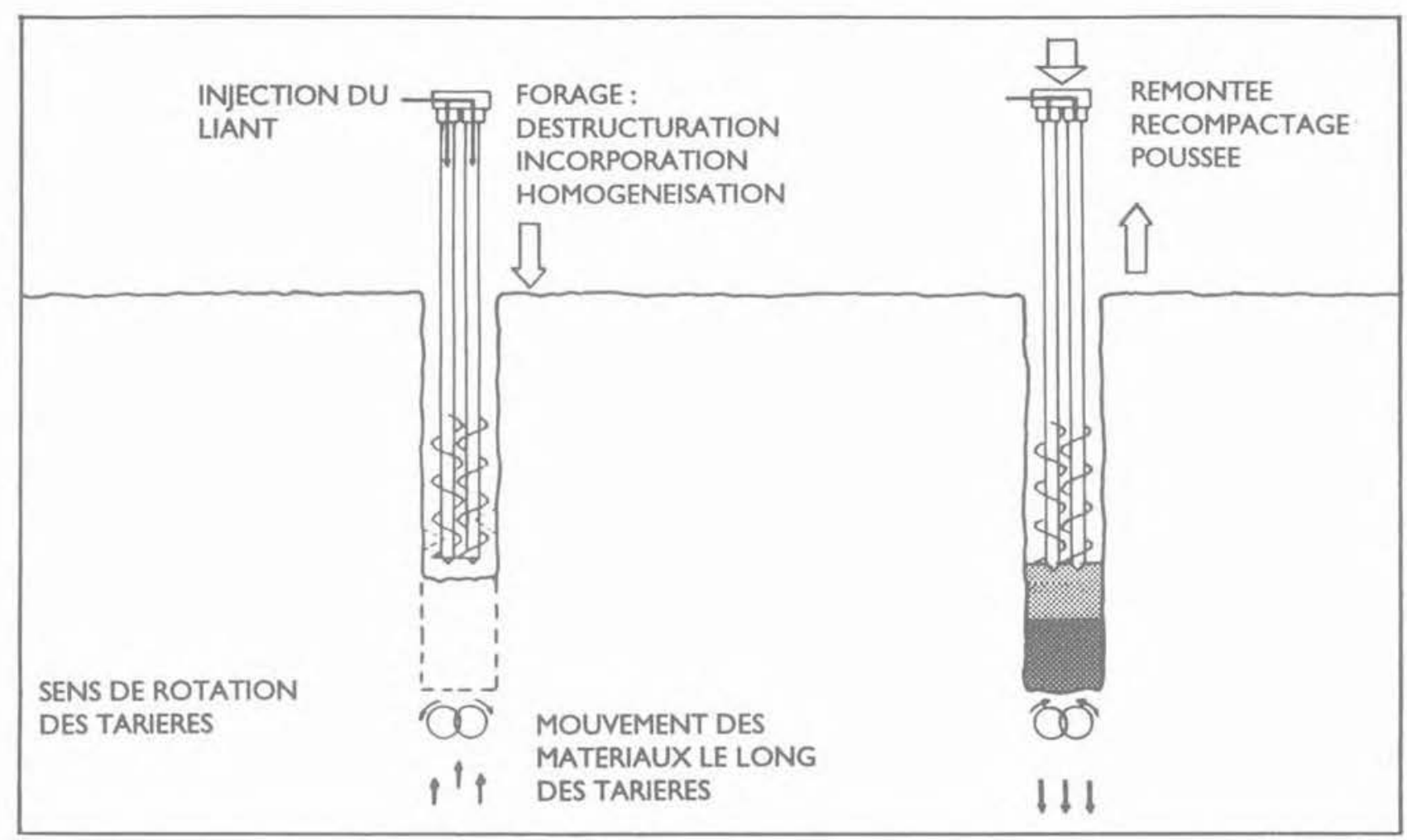

Fig. 9. - Procédé col mix.

Fig. 9. - Col mix process.

Il fait appel aux trois phénomènes distincts suivants intervenant indépendamment ou en combinaison ;

- une déstructuration fine du terrain en place sous l'effet mécanique d'un outil désagrégateur, renforcé par l'action physico-chimique d'un apport de chaux dans le cas de terrain argileux;

- une incorporation intime d'un liant de nature, dosage et degré d'hydratation adaptés aux caractéristiques du terrain traité et aux résultats recherchés; - un recompactage du mélange ainsi réalisé de façon à, au minimum, reconstituer et souvent améliorer la compacité du terrain existant avant traitement.

Ce procédé s'apparente aux procédés suédois de colonne à chaux et japonais de * deep-mixing *. II s'en distinge cependant fondamentalement :

- par le degré et la finesse d'homogénéisation du mélange terrain-liant d'apport ;

- par la possibilité de recompactage du mélange ainsi réalisé in situ.

Il est fondé sur l'utilisation d'un outil spécial constitué principalement de deux éléments de tarière de pas opposé, tournant en sens inverse et s'engageant l'un l'autre, leur extraxe étant inférieur à leur diamètre.

Cette " double tarière " est entraînée par un double moteur de forage à arbres creux transmettant un double mouvement de rotation inverse synchronisé à un double train de tiges creuses.

La rotation inverse des spirales emboitées de la double tarière provoque un phénomène de circulation longitudinale des matériaux.
Chaque élément de tarière porte à son extrémité inférieure un outil désagrégateur muni d'un ou plusieurs orifices en communication avec le train de tiges correspondant.

\subsection{Résumé}

Ces différentes techniques sont parfois utilisées en combinaison ou en complément d'autres techniques de traitements telles que le lavage du sol ou le traitement bactériologique.

Leur adaptation au domaine de l'environnemnt s'effectue sur la base d'une expérience acquise dans des domaines traditionnels en géotechnique. Elle nécessite en revanche une nouvelle approche en ce qui concerne les matériaux à utiliser, plus particulièrement au niveau de leur durabilité. On s'est donc attaché à mettre au point de nouvelles formulations de coulis destinées à la réalisation de coupures étanches pour le confinement de zones polluées.

\section{LES MATÉRIAUX DE PAROI}

\subsection{Présentation générale}

La paroi d'étanchéité moulée dans le sol constitue un procédé très largement utilisé dans le domaine des ouvrages hydrauliques.

Par extension des solutions pratiquées pour ces ouvrages, les écrans d'étanchéité sont très généralement réalisés à partir de coulis, de mortiers ou de bétons d'étanchéité. Les coulis sont composés d'argile, plus particulièrement de bentonite, et d'un liant hydrauli- 
que qui est généralement un ciment. Une charge inerte ou réactive peut être éventuellement rajoutée.

Les mortiers et bétons comportent les mêmes types de matériaux que les coulis avec, en plus, une quantité importante de granulats. Ils constituent un squelette granulaire, dont le fuseau est adapté entre autre pour assurer une compacité optimale. Lorsqu'ils sont inclus dans des ouvrages hydrauliques, ces matériaux sont soumis à une percolation d'eau relativement peu agressive à l'égard des ciments.

L'extrême diversité des sources potentielles de pollution rend impossible l'établissement d'une liste exhaustive de produits susceptibles d'être présents accidentellement dans les sols. On peut citer les catégories suivantes: acides, bases, métaux lourds, solvants, hydrocarbures, etc. pouvant être rencontrés à des concentrations extrêmement variées.

Certains de ces produits remettent en cause la durabilité des matériaux de composition des parois et par voie de conséquence les performances des ouvrages.

On peut, par exemple, souligner l'effet de différents produits organiques sur la perméabilité d'une argile illustré par la figure 10 .

On remarque que lorsque l'argile est percolée par de l'eau, son coefficient de perméabilité est de l'ordre de $10^{-8} \mathrm{~cm} / \mathrm{s}$. Ce coefficient augmente de plusieurs puissances quand l'argile est soumise à la percolation de produits tels que des alcools ou des hydrocarbures. Ce phénomène est du notamment à des modifications des distances inter-lamellaires de l'argile.

Par ailleurs, il est parfaitement connu que les acides concentrés $(\mathrm{pH}<5)$ affectent fortement la durabilité des ciments. Un coulis de ciment immergé dans une solution de $\mathrm{H}_{2} \mathrm{SO}_{4}$ à $\mathrm{pH} 1$ peut subir une perte en poids supérieure à $50 \%$ en 28 jours.

Ces variations sont fortement préjudiciables à des matériaux d'écran d'étanchéité. La dégradation s'effectue par contact soit à cause du gradient hydraulique, soit à cause de phénomènes de diffusion.

Une adaptation de la composition des coulis de paroi est donc nécessaire quand leur application sort du domaine "purement hydraulique » pour s'étendre à des confinements de zones renfermant des produits chimiques variés.

\subsection{Critères de durabilité des coulis argile-ciment}

La durabilité d'un coulis composé d'un liant hydraulique et d'argile est régie par le comportement du liant et par celui de l'argile.

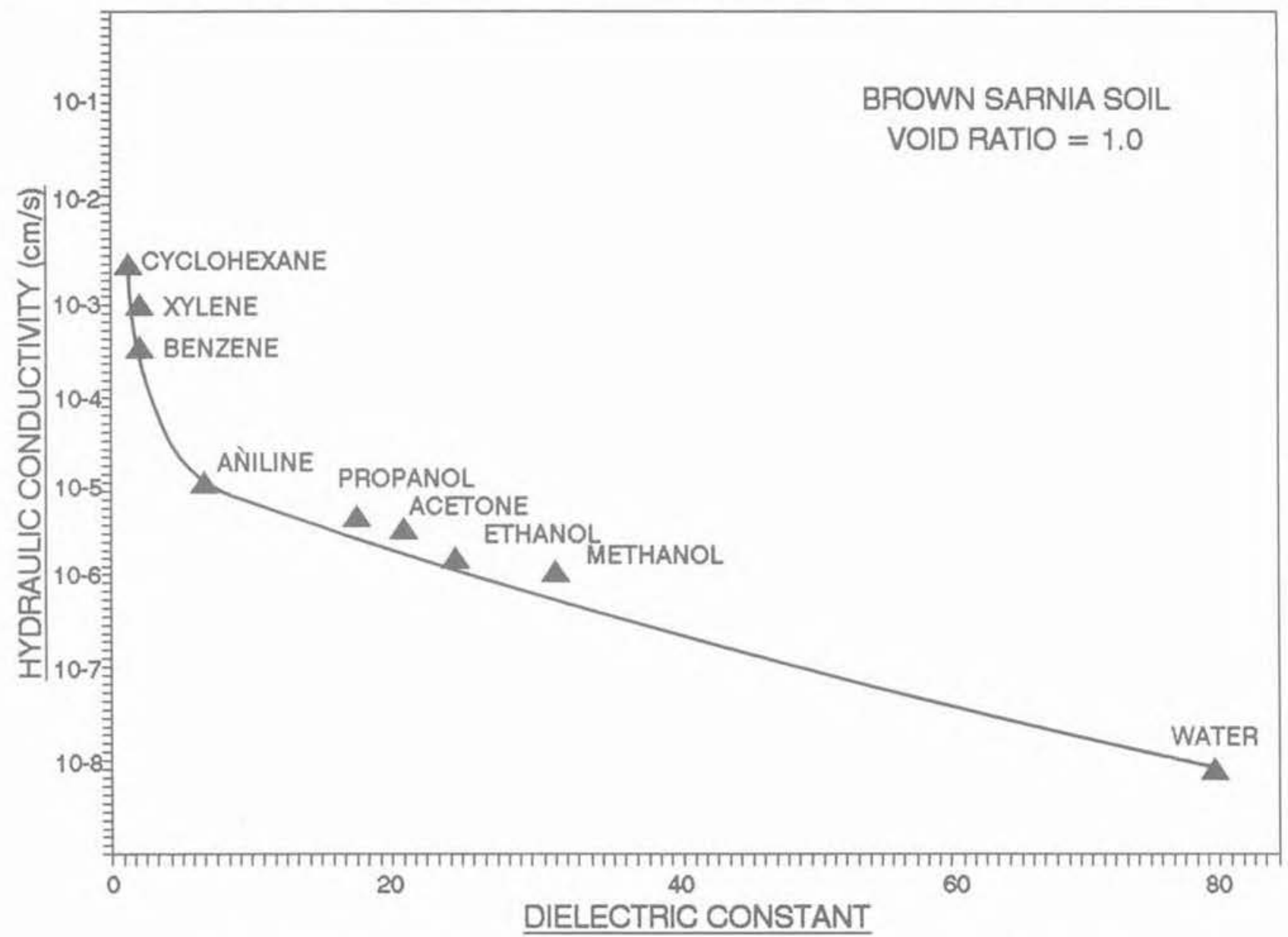

Fig. 10. - Conductivité hydraulique en fonction de la constante diélectique pour un indice de vide (d'après F. FERNANDEZ et R.M. QUIGLEY).

Fig. 10. - Hydraulic conductivity vs dielectric constant for a void ratio of unity (ref. F. FERNANDEZ and R.M. OUIGLEY). 
L'adaptation de leur dosage et le choix du type de matériau permet jusqu'à un certain degré d'agressivité de formuler des compositions stables et pérennes en augmentant la résistance intrinsèque du coulis.

D'autre part, la limitation du transport des produits à l'intérieur du coulis est obtenue en abaissant le coefficient de perméabilité du mélange après prise.

Les deux critères :

- stabilité intrinsèque des matériaux ;

- faible coefficient de perméabilité :

conduisent à l'établissement d'un tableau de * classification "en fonction du degré d'agressivité des produits.

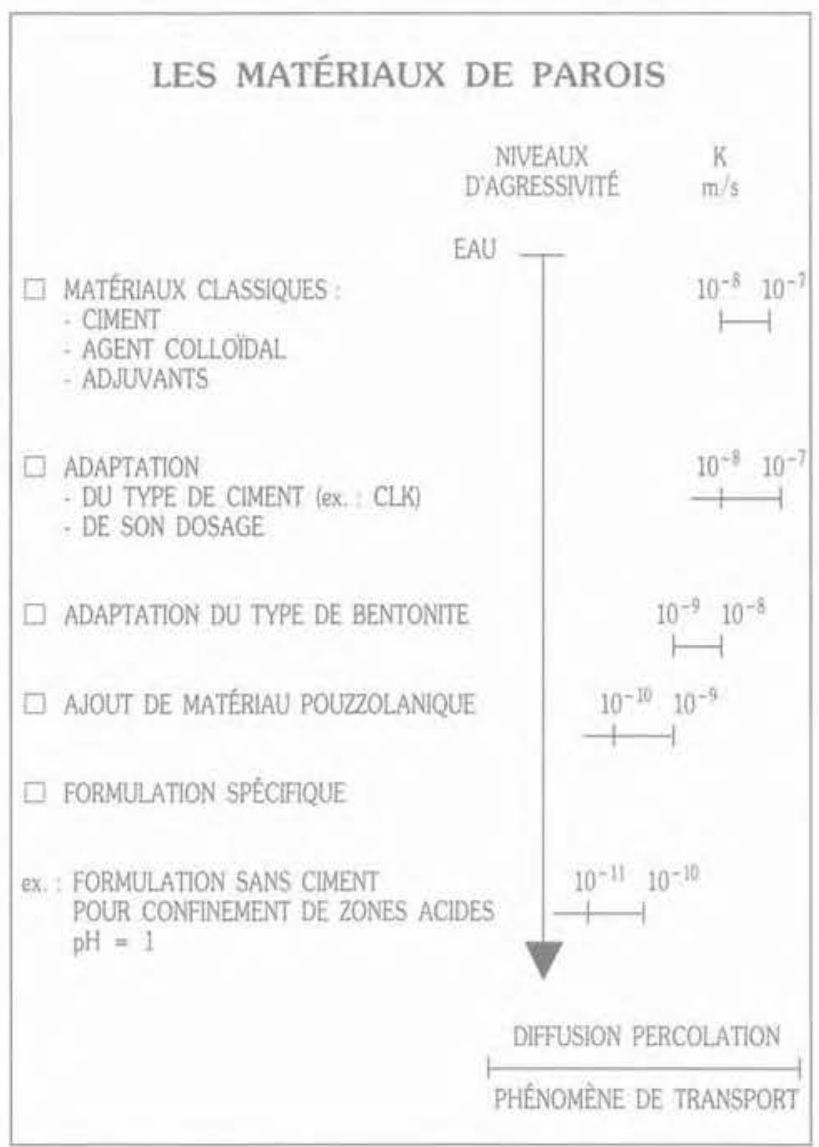

Fig. 11. - Classification des matériaux de parois.

Fig. 11. - Classification of slurry walls materials.

Il est possible de cette façon de formuler des coulis à base d'argile et de ciment stables dans des milieux de natures chimiques et de concentrations variables.

Un exemple d'adaptation de composition d'un coulis à la tenue d'un lixiviat de synthèse est illustré par la figure 12 .

Un lixiviat a été reproduit sur la base d'une composition réelle d'un lixiviat provenant d'une décharge de déchets industriels. Ses caractéristiques physicochimiques étaient les suivantes :

$-\mathrm{pH}=6,7$

- $\mathrm{DCO}=14700 \mathrm{mg} / \mathrm{l}$

- conductivité $=28900 \mu \mathrm{S} \mathrm{cm}^{-1}$
$-\mathrm{COT}=6340 \mathrm{mg} / \mathrm{l}$

$-\mathrm{Eh}=-18 \mathrm{mV}$.

Après immersion dans ce lixiviat (réf. Norme AST C267-82) des échantillons de formulation classique subissent une perte en poids supérieure à $5 \%$ au bout de 50 jours.

Dans des conditions de tests idendiques, les variations pondérales de ces coulis immergés dans l'eau ne sont que de $\pm 1 \%$ (voir fig. 13).

$\mathrm{Ce}$ lixiviat est donc particulièrement agressif.

L'addition de matériaux spécifiques permet d'obtenir des compositions répondant au critère de durabilité recherchée.

Leur variation pondérale en cours d'immersion n'est plus que de $\pm 1 \%$, ce qui correspond aux valeurs observées pour des coulis stockés dans l'eau. Ce test de durabilité est particulièrement sévère mais permet de se placer dans des conditions de sécurité optimale.

Il est ainsi possible d'adapter la composition de coulis argile-ciment jusqu'à un certain degré d'agressivité.

On peut citer à titre d'exemples parmi les produits affectant la durabilité de coulis classiques et pour lesquels une telle adaptation est possible:

- des pH acides jusqu'à environ 4;

- des teneurs en sels d'ammonium supérieures à $100 \mathrm{mg} / 1$;

- des pH très alcalins (14).

Ces formulations répondent à des critères de durabilité exprimés en termes de stabilité pondérale et de perméabilité en plus de spécifications rhéologiques et mécaniques.

\subsection{Béton plastique résistant aux acides}

Comme mentionné au paragraphe précédent, une adaptation de la composition d'un coulis à base de ciment peut être réalisée pour résister à des $\mathrm{pH}$ acides de l'ordre de 4.

Pour des acides plus concentrés, l'utilisation de ciment est fortement déconseillée.

L'attaque acide se déroule en deux étapes:

- neutralisaltion de la chaux libre et de la Portlandite ;

- hydrolyse des sels de calcium à caractère basique.

Cette hydrolyse peut continuer jusqu'à la décalcification complète plus ou moins rapidement selon la force de lacide. Il a donc fallu faire une adaptation de composition d'un béton plastique résistant à des percolations acides.

\subsubsection{Approche expérimentale}

Le squelette granulaire présente des caractéristiques de répartition granulométrique telle qu'il soit autofiltrant et que, notamment, le matériau argileux ne risque pas d'être entraîné par le courant de percolation. Cette caractéristique est obtenue par incorporation, en quantité appropriée, de matériaux fins de granulomé- 
$\%$ Variation pondérale

$\%$ Weight change

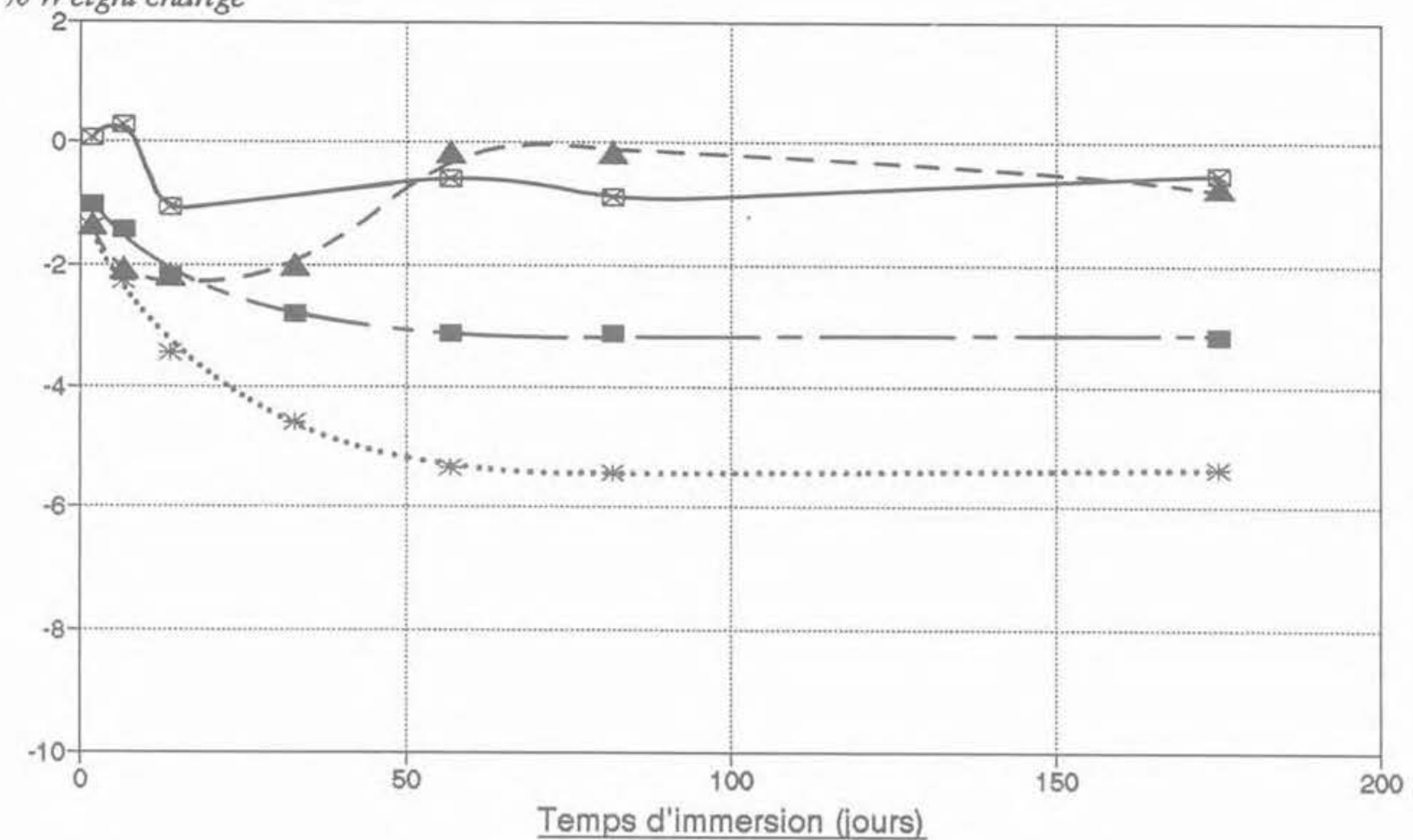

Time (days)

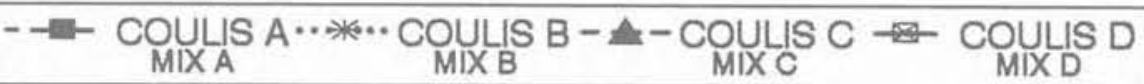

Fig. 12. - Test d'immersion/Lixiviat de synthèse. - Fig. 12. - Immersion test/Synthetic leachate.

\% Variation pondérale

$\%$ Weight change

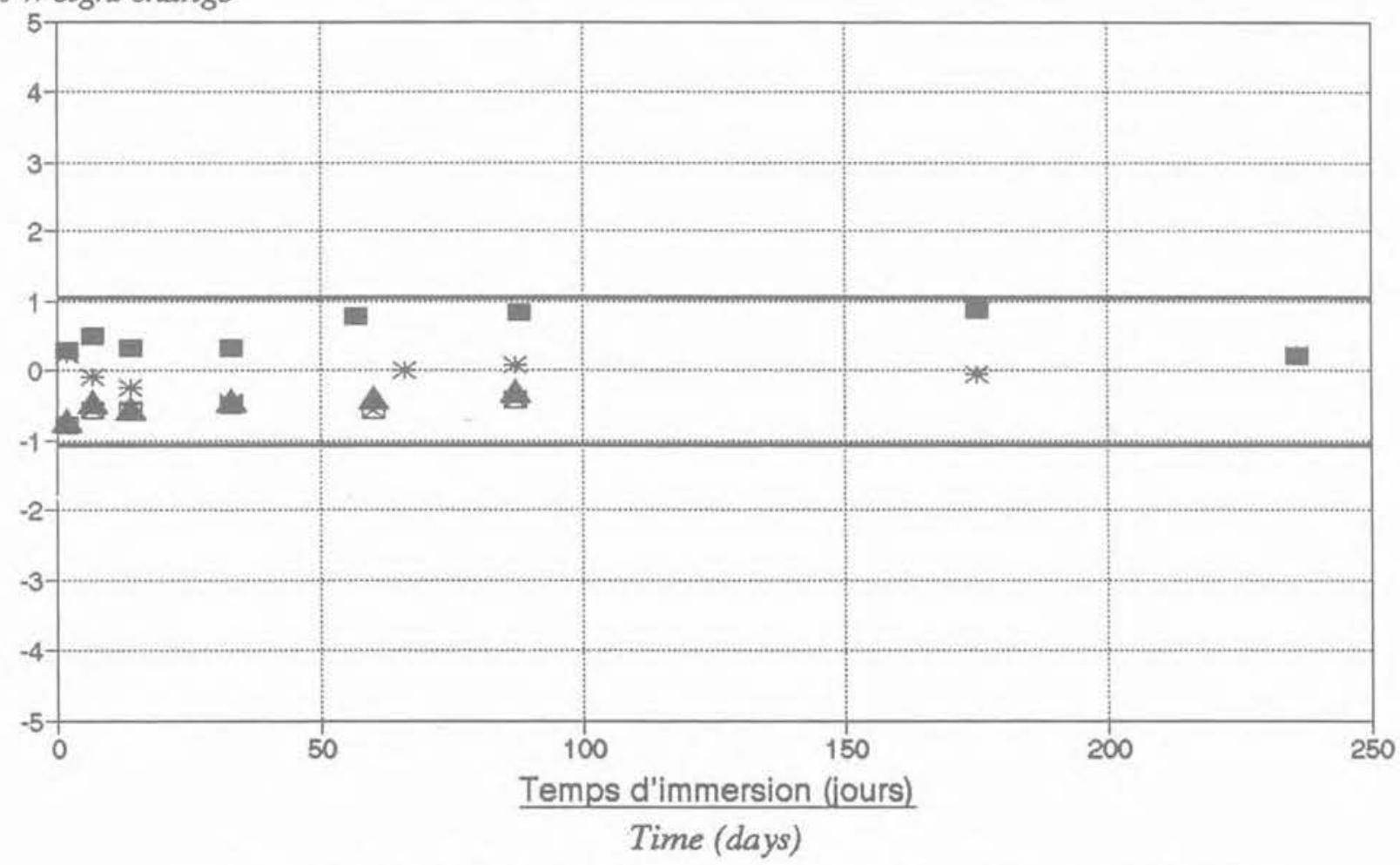

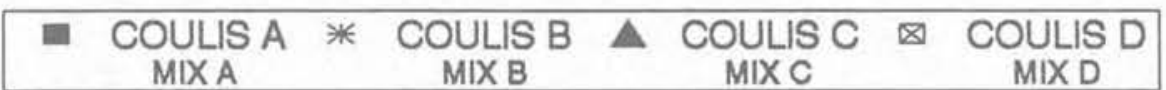

Fig. 13. - Test d'immersion/Eau. - Fig. 13. - Immersion test/water. 
trie comprise entre $0,002 \mathrm{~mm}$ et $0,1 \mathrm{~mm}$. Il est constitué de matériaux siliceux choisis pour leur résistance en milieu acide. Des granulats calcaires seraient, en effet, entièrement détruits.

Le dosage du matériau argileux tel que la bentonite est ajusté en fonction de la perméabilité requise.

Des essais de filtration permettent d'optimiser les dosages (fig. 14 et 15).

Une telle formulation, granulats + argiles + fines, permet d'obtenir un béton plastique de bonne étanchéité.

Pour déterminer de façon accélérée le comportement des bétons d'argile soumis à une percolation d'eau très acide, des essais de perméabilité sous gradient élevé $(i=25$ ou 330$)$ sont réalisés avec des eaux dont le $\mathrm{pH}$ est amené aux valeurs de 1 et 0 par addition d'acide sulfurique ou d'acide phosphorique. L'évolution du débit de percolation d'une solution à $\mathrm{pH}=$ 1 à travers un béton dont le matériau argileux est une bentonite sodique est illustrée (fig. 16).

On constate une augmentation rapide de la perméabilité après un délai qui correspond, pour les conditions d'essais, à la déstructuration de la bentonite par l'acide. Cette expérience permet de déterminer la quantité critique d'ions acides qui entraînent la dégradation du dosage étudié.

Une méthode originale a été mise au point qui permet de protéger les bétons d'étanchéité à base d'argile contre la dégradation qu'entraine la percolation d'eaux très acides. Elle consiste à incorporer un silicate alcalin comme constituant additionnel de la composition des bétons.

Quand un béton d'étanchéité pour paroi moulée, préparé avec incorporation d'un silicate alcalin, est soumis à une percolation d'eau acide, celle-ci, en péné-

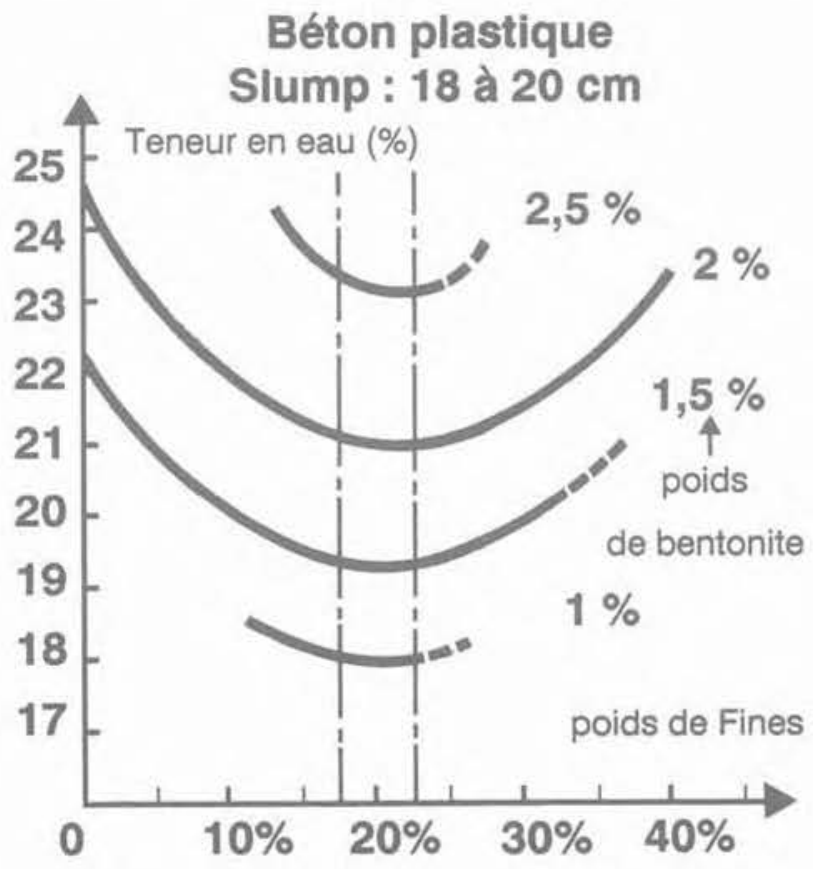

Fig. 14. - Teneur en eau du béton plastique en fonction du pourcentage de fines et de bentonite.

Fig. 14. - Water content of plastic concrete, vs percentage of fines and bentonite.

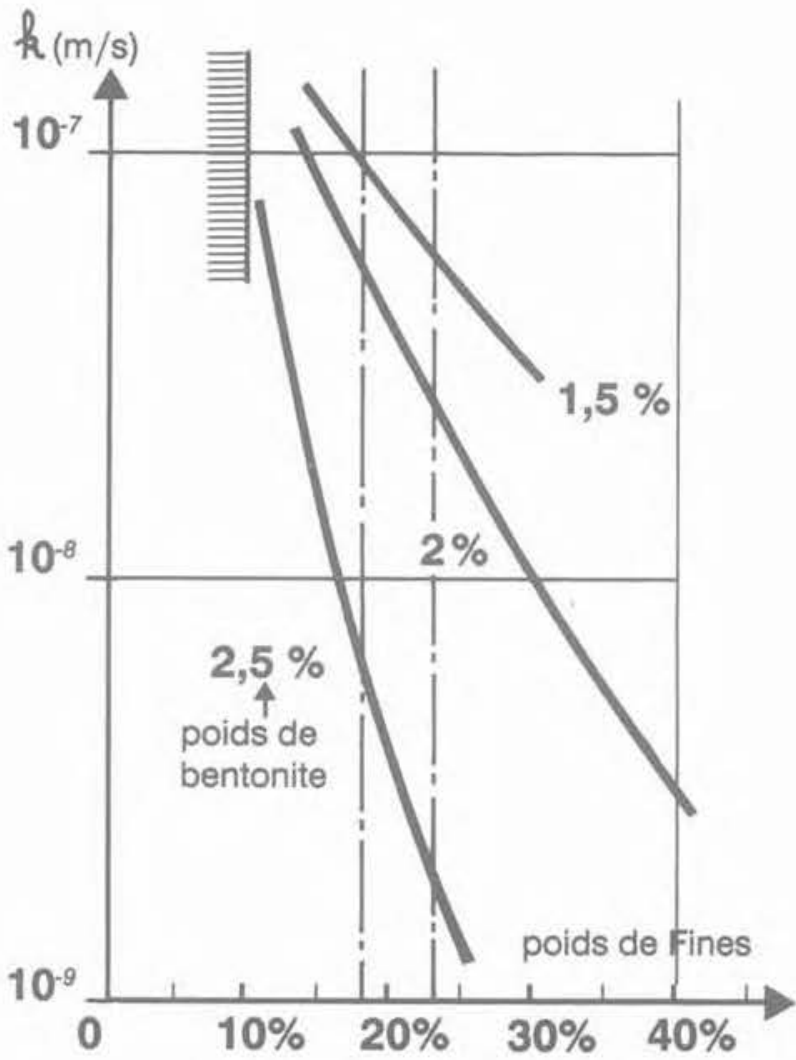

Fig. 15. - Perméabilité du béton plastique en fonction du pourcentage de fines et de bentonite.

Fig. 15. - Permeability of plastic concrete vs percentage of fines and bentonite.

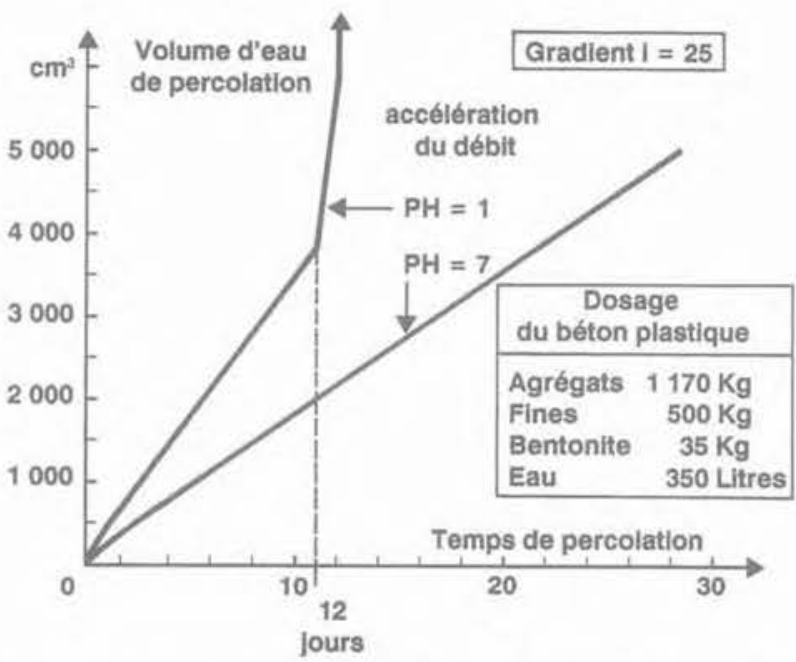

Fig. 16. - Essais de perméabilité à la cellule triaxiale. Effet de l'acidité de l'eau.

Fig. 16. - Triaxial cell permeability test. Effect of water acidity.

trant, provoque une neutralisation de la soude ou de la potasse qui entraîne une précipitation de silice amorphe dans les pores du béton. Cette silice est insoluble en milieu acide. Il en résulte une amélioration considérable et durable de l'étanchéité aux eaux acides. L'effet de la percolation de solutions très acides sur la perméabilité de bétons plastiques est illustré (fig. 17). Une solution de $\mathrm{pH}=0$ percolant à travers un 


\section{Essais de perméabilité au filtre presse Gradient I $=330$}

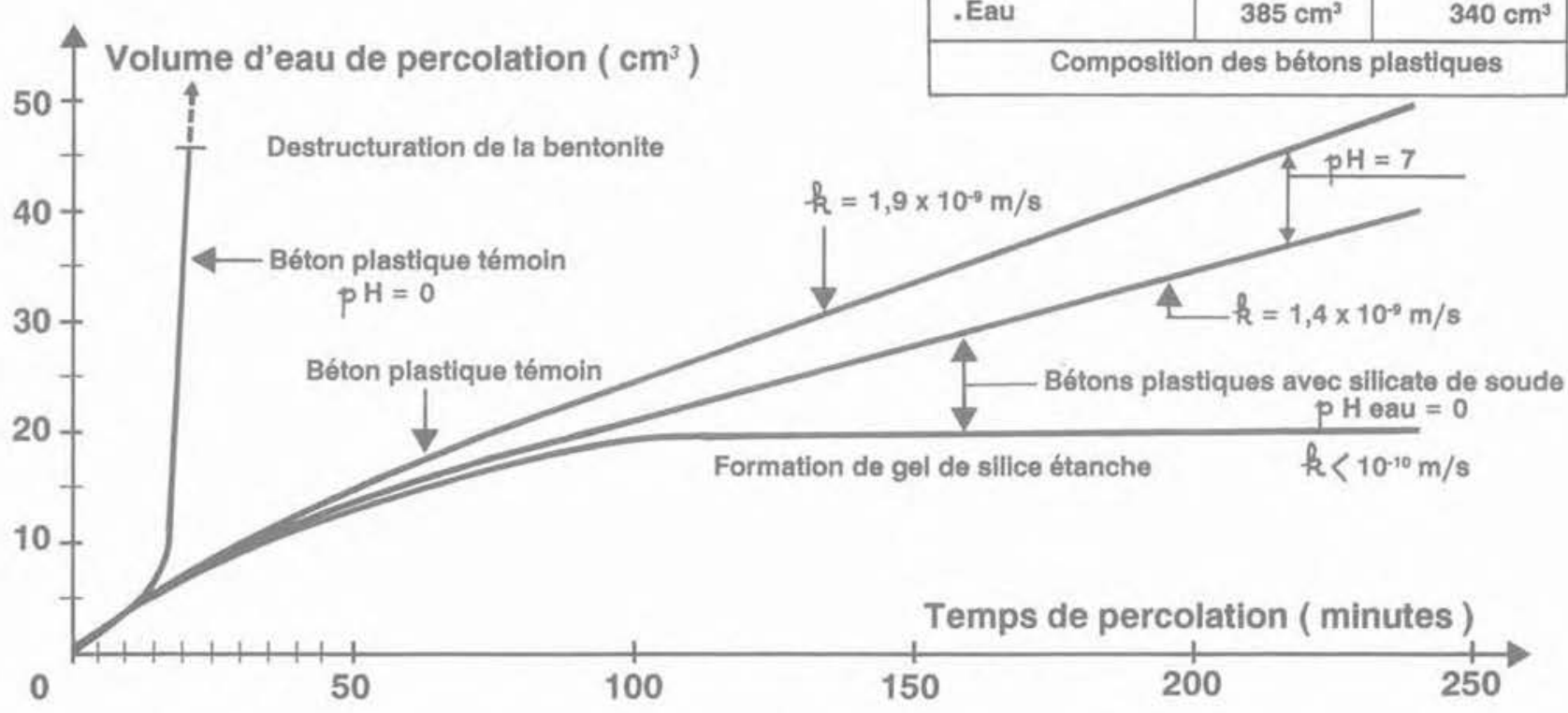

Fig. 17. - Influence de l'acidité sur la perméabilité du béton plastique. Effet du silicate de soude. Fig. 17. - Influence of acidity on the permeability of plastic concrete. Effect of sodium silicate.

béton préparé avec un silicate alcalin entraîne une amélioration de l'imperméabilité puisque la valeur atteint rapidement un niveau inférieur à $1.10^{-10}$ $\mathrm{m} / \mathrm{s}$.

\subsubsection{Exemple d'application}

Le complexe pétrochimique de Moron, situé en bord de mer à $225 \mathrm{~km}$ à l'est de Caracas présente une zone de dépôt de déchets industriels dont la caractéristique principale est une forte teneur en mercure.

Un confinement de ce dépôt a été terminé en janvier 1988 par la réalisation d'un écran d'étanchéité ceinturant la zone contaminée. L'écran rejoint un horizon profond étanche et isole le dépôt de mercure de l'aquifère supérieur qui est soumis à un gradient d'écoulement vers la mer.

Cet ouvrage schématisé (fig. 18) représente $8000 \mathrm{~m}^{2}$ d'écran réalisé avec une épaisseur de $0,60 \mathrm{~m}$. Il a la particularité d'être localisé à l'aval d'une usine de fabrication d'acides. Des effluents chargés en acide phosphorique et acide sulfurique, caractérisés par un $\mathrm{pH}$ éventuellement inférieur à 2 , peuvent venir au contact de la paroi. Celle-ci a été réalisée à l'aide d'un béton plastique d'étanchéité composé de granulats et de sable siliceux, de fines siliceuses qui permettent d'obtenir le squelette granulaire à répartition continue de la figure 19 , de bentonite et de silicate.

Caractéristiques du béton :

Densité : 1,95

Kc initial : 2 à $7 / 10^{-10} \mathrm{~m} / \mathrm{s}$

Kc final : $<1.10^{-10} \mathrm{~m} / \mathrm{s}$

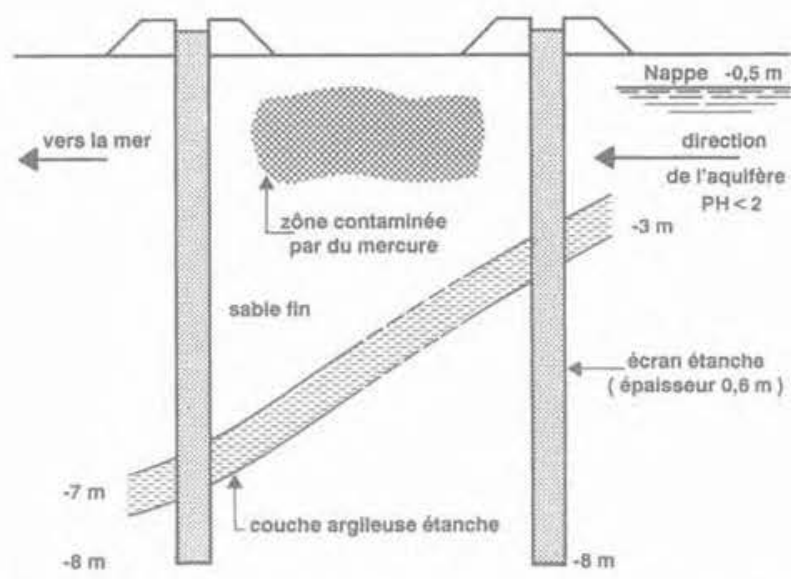

Fig. 18. - Confinement par un écran en béton plastique de la zone de terrain contaminé.

Fig. 18. - Containment of the contaminated area by plastic concrete wall.

\section{PAROI AVEC INCORPORATION D'UNE MEMBRANE PLASTIQUE}

\subsection{Description}

Une paroi verticale réalisée en béton plastique peut supporter certains mouvements de terrain sans risque de fissuration ou de rupture. En revanche, quand de grands mouvements de terrains sont à craindre, une telle paroi peut ne pas présenter une plasticité suffisante. Cette propriété, très importante dans les ouvrages hydrauliques classiques, devient primordiale quand il s'agit de confinement de déchets industriels. 


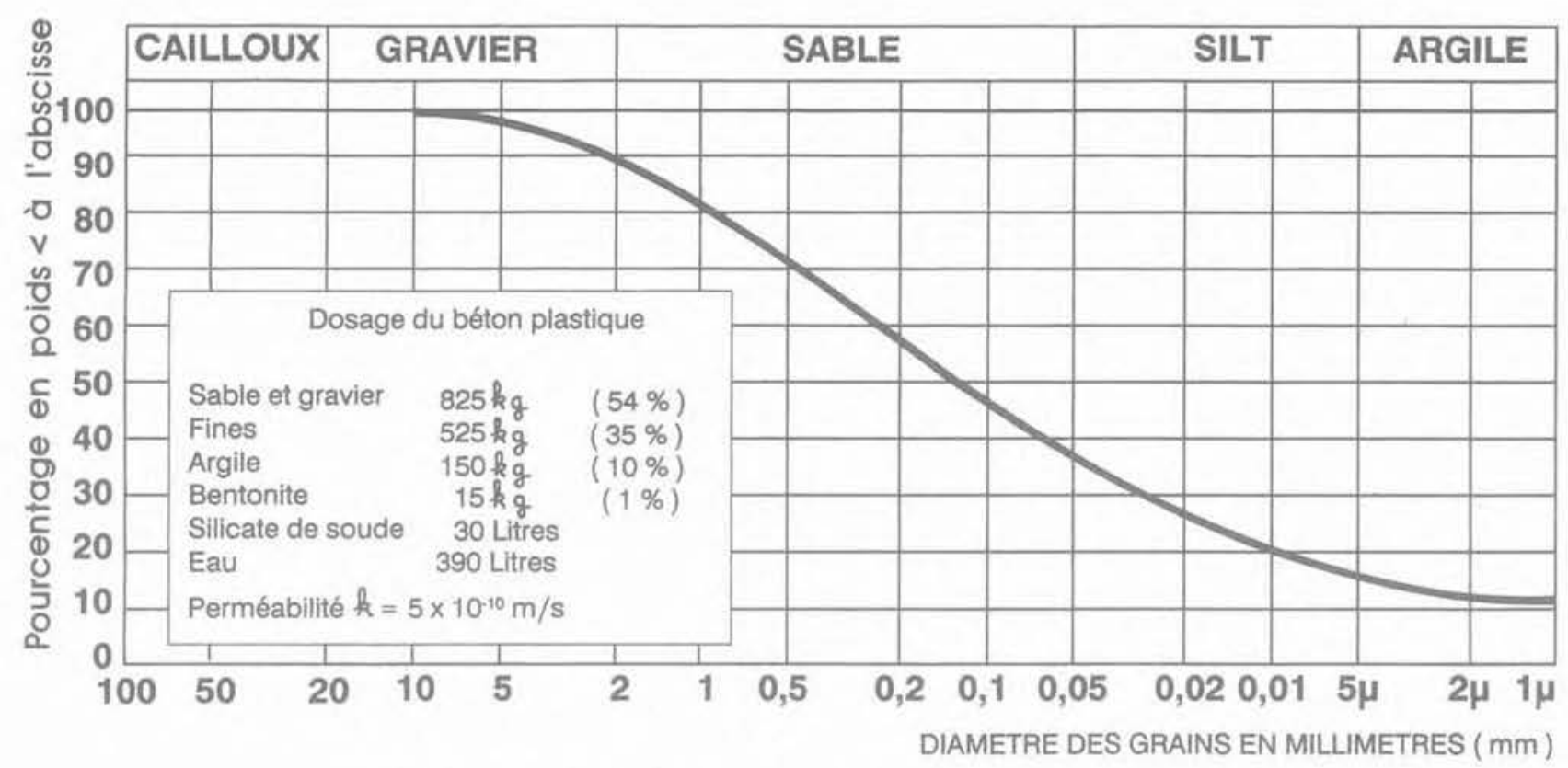

Fig. 19. - MORON - Analyse granulométrique du béton plastique.

Fig. 19. - MORON - Grain size distribution of plastic concret.

Une solution consiste à incorporer une feuille plastique au milieu d'une paroi au coulis. Une technique repose sur l'utilisation d'une membrane GEOLOCK. Sa mise en place est maîtrisée à des profondeurs allant jusqu'à $30 \mathrm{~m}$.

GEOLOCK est un écran plastique constitué de palfeuilles en polyéthylène haute densité (PEHD) assemblées par un système de serrure qui forme un joint étanche prévenant toute fuite due à la perméabilité du sol contaminé,

Une propriété intéressante de cet assemblage est de rendre l'écran de palfeuille parfaitement étanche en introduisant un joint Néoprène expansif résistant aux produits chimiques, dans un logement pratiqué à l'intérieur de l'élément femelle.

La capacité de dilatation du joint dans l'eau permet d'atteindre un volume seize fois supérieur au volume initial.

Les membranes en PEHD sont reconnues pour leur inertie à l'égard d'une grande variété de produits chimiques.

L'imperméabilité au gaz de ce système a été démontrée pour des pressions allant jusqu'à 7 bar.

\subsection{Exemples d'application}

Une application illustre l'utilisation du système GEOLOCK dans le cadre de la protection de l'environnement sur le site de la centrale thermique de Castle Peak à Hong-Kong. Cette centrale utilise du charbon et génère des quantités importantes de cendres volan-

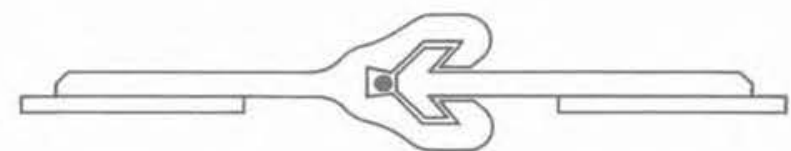

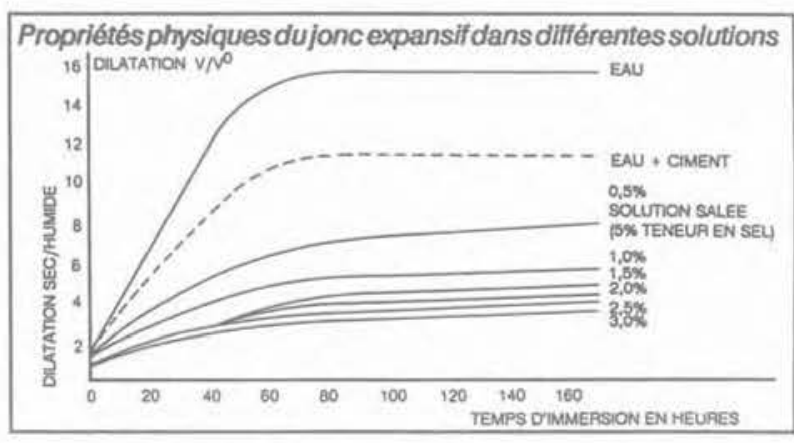

Fig. 21. - Expansion du joint hydrotite dans différentes solutions.

Fig. 21. - Expansion of hydrotite lock in some mineral solutions.

tes qui sont stockées par voie humide dans des lagons aménagés en bordure de la Deep Bay. Cette baie réputée pour ses parcs à huîtres et des précautions particulières sont prises pour éviter toute pollution de l'eau de mer par les eaux d'essorage des cendres renfermant des métaux lourds.

La protection comporte une digue étanchée par une paroi d'étanchéité au coulis de $1000 \mathrm{ml}$ de long. ancrée de $200 \mathrm{~mm}$ dans le rocher sous-jacent.

La membrane est mise en place au milieu de la paroi au coulis.

Un autre exemple concerne la protection d'une zone d'habitation contre la migration de gaz méthane provenant d'un dépôt de déchets.

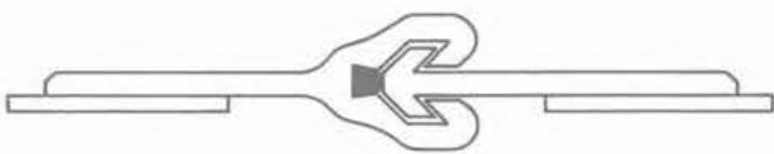

Fig. 20. - Système GEOLOCK - Jonc expansif avant et après dilatation.

Fig. 20 - GEOLOCK - Expansion profile before and after dilatation. 


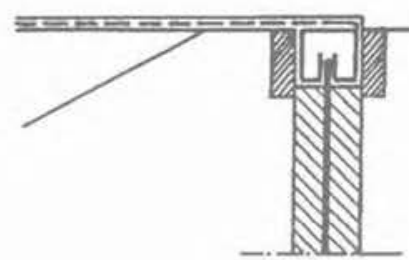

DETAILA

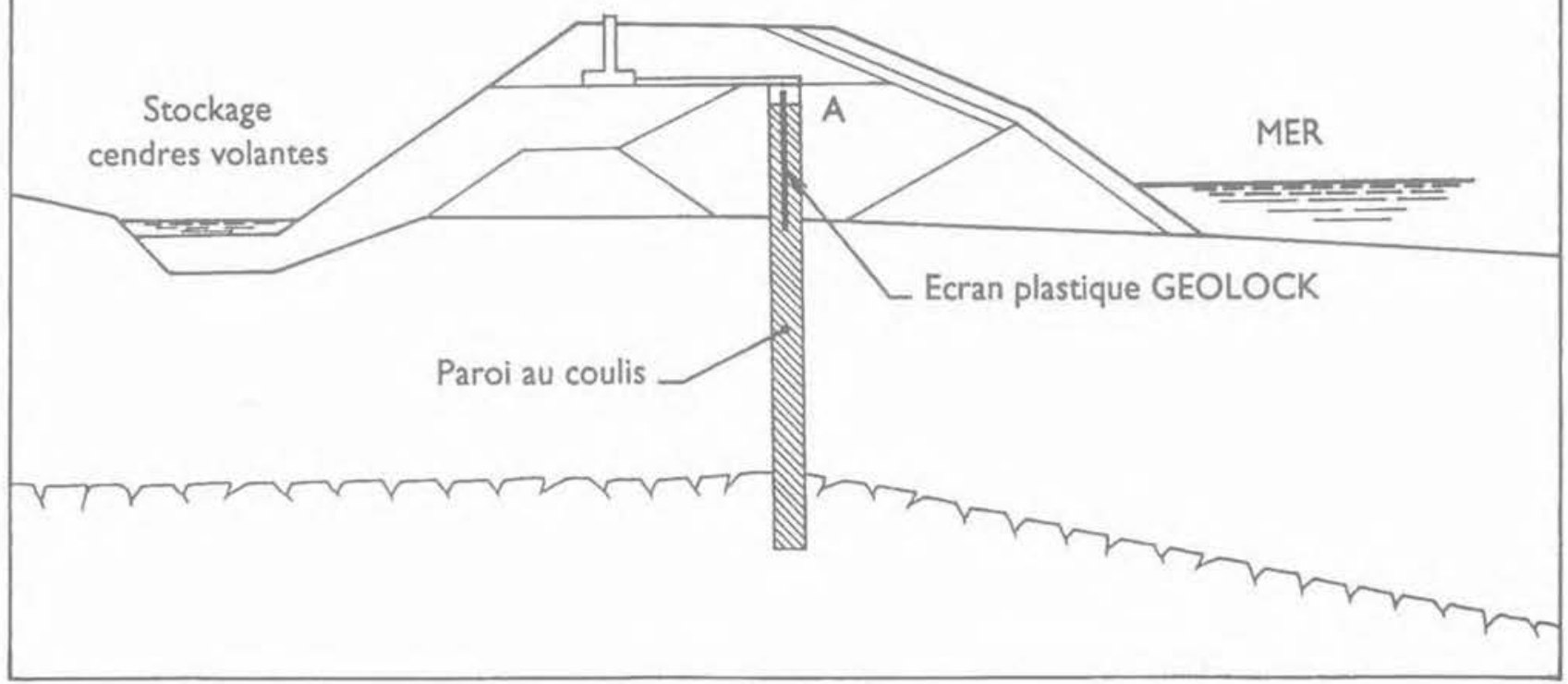

Fig. 22. - GEOLOCK - Castle Peak - Hong-Kong.

Fig. 22. - GEOLOCK - Castle Peak - Hong-Kong.

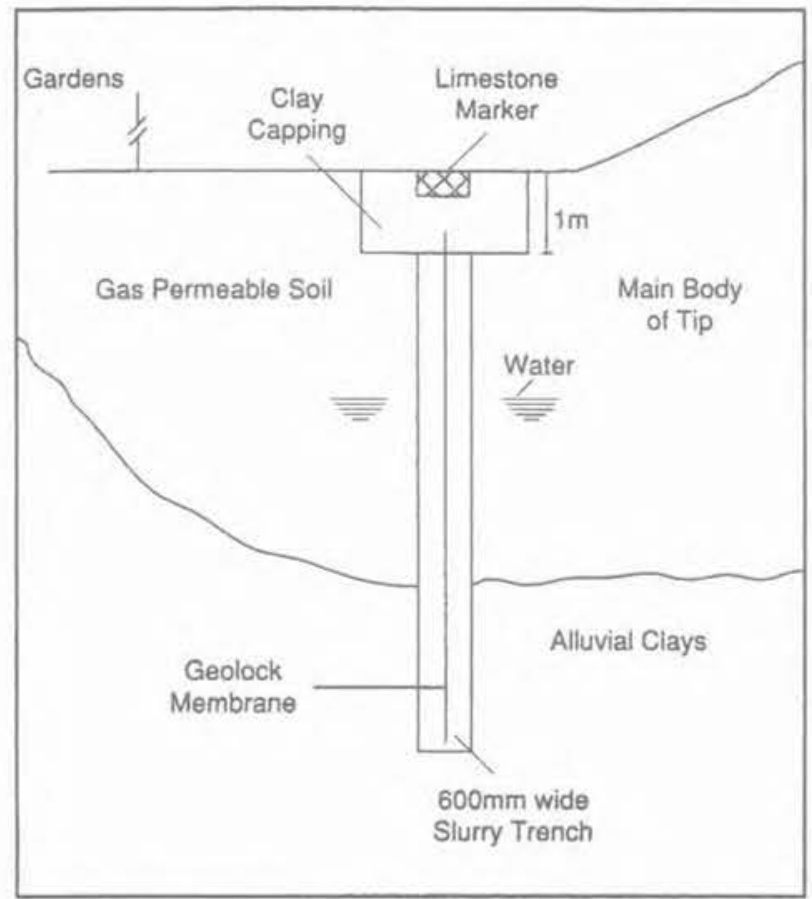

Fig. 23. - IRLAM GB - Coupe paroi aU GEOLOCK. Fig. 23. - IRLAM UK - Typical cross-section through cut-off wall.

\section{CONCLUSION}

Sur la base d'une expérience acquise dans des domajnes traditionnels, des techniques et des matériaux ré- pondant au souci de protection et sauvegarde de lenvironnement ont été mis au point ces dernières années.

Ceci a impliqué une nouvelle approche au niveau de leur conception et de leur mise en cuvre.

La poursuite des recherches dans ce domaine est réalisée en intégrant l'évolution de systèmes de réglementation dans le but d'apporter de nouvelles solutions au problème de pollution de sols et d'aquifères.

\section{BIBLIOGRAPHIE}

GANDAIS M. (1985), Coulis d'injection à haute pénétrabilité : MICRON S et C3S. CFGB.

ASTM C267-82, Standard test method for chemical resistance of mortars, grouts and monolithics surfacings.

NF P18-011, Bétons classification des environnements agressifs.

BODOCSI et al. (1988), Reactivity of various grouts to hazardous wastes and leachates. EPA/600/2-88/021.

GANDAIS M. et ESNAULT A. (1990), Ecrans d'étanchéité appliqués au confinement de déchets indus triels, Colloque *Géotechnique et Environnement », 4e Entretiens du Centre Jacques-Cartier, Grenoble. 